\title{
Effets De La Fertilisation Sur Les Nématodes Parasites Et Le Rendement En Rhizomes Frais Du Gingembre, Zingiber officinale Rosc
}

\author{
Assih Alèdi \\ Laboratoire de recherche sur les agroresssources et la santé \\ environnementale, Ecole Supérieure d'Agronomie, Université de Lomé, \\ Togo \\ Institut Togolais de Recherche Agronomique (ITRA), Togo \\ Nenonene Yawo Amen \\ Laboratoire de recherche sur les agroresssources et la santé \\ environnementale, Ecole Supérieure d'Agronomie, Université de Lomé, \\ Togo

\section{Tchabi Atti} \\ Institut Supérieur des Métiers de l'Agriculture, Université de Kara, Togo

\section{Fiaboe Kokou Rodrigue} \\ Ecole Supérieure d'Agronomie, Université de Lomé, Togo \\ Akantetou Komla Pikassalé \\ Institut Togolais de Recherche Agronomique (ITRA), Togo
}

Doi: 10.19044/esj.2018.v14n24p216 URL:http://dx.doi.org/10.19044/esj.2018.v14n24p216

\section{Abstract}

Ginger, Zingiber officinale is one of the most cultivated spices in the world. Therefore its production is often subject to high pest and disease pressures, particularly that of plant parasitic nematodes. For the sustainable management of parasitic nematodes of ginger, a trial was conducted at Agronomic Research Station of the Graduate School of Agronomy at the University of Lomé to assess the efficacy of some organo-mineral fertilizers on parasitic nematodes. An organic manure based on house waste valorization in three doses $\left(0\right.$ tha $^{-1} ; 15$ tha $^{-1}$ and 25 tha $\left.^{-1}\right)$ and five mineral fertilizer formulations from simple mineral fertilizers (Urea $46 \%$, the chloride of potassium and the triple superphosphate): $\mathrm{N}_{0} \mathrm{P}_{0} \mathrm{~K}_{0} ; \mathrm{N}_{45} \mathrm{P}_{15} \mathrm{~K}_{60} ; \mathrm{N}_{70} \mathrm{P}_{50} \mathrm{~K}_{50}$; $\mathrm{N}_{90} \mathrm{P}_{30} \mathrm{~K}_{100}$ and $\mathrm{N}_{125} \mathrm{P}_{90} \mathrm{~K}_{100}$ ) were used according to a split plot design. The inventory of the nematodes permitted to count five genders of nematodes: Meloidogyne; Xiphinema; Longidorus; Helicotylenchus and Pratylenchus. The results indicated that an input of 25 tons of organic manure associated with mineral fertilizer of $\mathrm{N}_{70} \mathrm{P}_{50} \mathrm{~K}_{50}$ and $\mathrm{N}_{125} \mathrm{P}_{90} \mathrm{~K}_{100}$ reduce nematode density 
by up to $58.62 \%$ in roots and $95.88 \%$ in soil compared to the treatment without manure and mineral fertilizer. The yield of fresh rhizomes vary from 2.9 to 7.7 t/ha with little statistically effects of the manures. This study reveals that nematodes constitute a serious phytosanitary problem for ginger and that the contribution of organo-mineral fertilization makes it possible to reduce the density of nematodes under ginger.

Keywords: Zingiber officinale, Fertilization, Nematodes, Yield.

\section{Résumé}

Le gingembre, Zingiber officinale, est l'une des épices les plus cultivées au monde. Cependant, sa production est souvent sujette à une forte pression parasitaire en particulier celle des nématodes phytoparasites. Pour une gestion durable de ces nématodes, un essai a été conduit à la Station d'Expérimentation de l'Ecole Supérieure d'Agronomie de l'Université de Lomé afin d'évaluer l'efficacité des engrais organo-mineraux sur les nématodes phytoparasites. Une fumure organique à base des ordures ménagères en trois doses $\left(0\right.$ tha $^{-1} ; 15$ tha $^{-1}$ et 25 tha $\left.^{-1}\right)$ et cinq formulations d'engrais minéraux à partir d'engrais minéraux simples (Urée 46\%, le chlorure de potassium et le triple superphosphate) : $\mathrm{N}_{0} \mathrm{P}_{0} \mathrm{~K}_{0} ; \mathrm{N}_{45} \mathrm{P}_{15} \mathrm{~K}_{60} ; \mathrm{N}_{70} \mathrm{P}_{50} \mathrm{~K}_{50}$; $\mathrm{N}_{90} \mathrm{P}_{30} \mathrm{~K}_{100}$ et $\mathrm{N}_{125} \mathrm{P}_{90} \mathrm{~K}_{100}$ ont été utilisées suivant un dispositif en split plot. L'inventaire des nématodes a permis de recenser cinq genres : Meloidogyne ; Xiphinema; Longidorus; Helicotylenchus et Pratylenchus. Les résultats ont montré qu'un apport de 25 tonnes de fumure organique associé aux formulations $\mathrm{N}_{70} \mathrm{P}_{50} \mathrm{~K}_{50}$ et $\mathrm{N}_{125} \mathrm{P}_{90} \mathrm{~K}_{100}$ de fumure minérale permet de réduire de façon significative la densité des populations de nématodes de 58,62\% dans les racines et $95,88 \%$ dans le sol de par rapport au témoin sans engrais. Les rendements en rhizomes frais obtenus ont varié de 2,9 à 7,7 t/ha avec des effets des fumures statistiquement peu marqués. Cette étude révèle que les nématodes constituent un sérieux problème phytosanitaire pour le gingembre et que l'apport de la fumure organo-minérale permet de réduire la densité des nématodes sous le gingembre.

Mots clés : Zingiber officinale, Fertilisation, Nématodes, Rendement.

\section{Introduction}

Le gingembre, Zingiber officinale Roscoe est une herbe annuelle de la famille des zingibéracées cultivée un peu partout dans la zone tropicale. Originaire d'Asie du sud-est (Preeti et al., 2008), le gingembre est cultivé essentiellement pour ses rhizomes. C'est l'une des plus importantes épices au monde de par son importance économique et ses nombreuses vertus médicinales (Nandkangre, 2015). Les nombreuses vertus médicinales 
(amélioration de la qualité du sperme, protection de l'Acide Désoxyribonucléique, anticoagulant, anti-hypercholestérolémie, antihyperglycémiant, antispasmodique, antimicrobien, etc.) sont dues à deux de ses constituants : l'oléorésine et l'huile essentiel (Eleazu et al., 2013). La production du gingembre est souvent limitée à causes de la pression parasitaire notamment celle des nématodes (Koshy et al., 2005) et d'une fertilisation inadaptée (Egbuchua \& Enujeke, 2013). Contrairement aux insectes nuisibles et maladies qui bénéficient d'une grande attention, peu de travaux de recherche ont été menés sur le contrôle durable et efficace des nématodes sur cette culture. Les nématodes, en raison de la grande diversité de leurs hôtes, de leur cycle de reproduction très court, de leur capacité de reproduction très élevée et de leur nature endoparasitique (Trudgill \& Blok, 2001; ManzanillaLopez et al., 2004) sont difficiles à combattre. Présents sous toutes les latitudes; ils représentent un grave problème phytosanitaire, surtout dans le monde tropical où règne en permanence un climat favorable à leur multiplication (Traore, 2012). Au Togo, comme dans les autres pays de l'Afrique de l'Ouest, la lutte chimique est la méthode la plus utilisée par les producteurs pour la protection des nuisibles des cultures, notamment les cultures maraîchères (James et al., 2010). Mais, le coût élevé des nématicides de synthèse et les risques liés à leur utilisation nécessitent la recherche de méthodes alternatives permettant une protection saine et durable de la culture tel que les amendements organiques (El Badri et al., 2008).

La présente étude se donne pour objectif de développer une méthode de lutte intégrant l'utilisation d'engrais organique et d'engrais minéraux simples afin d'assurer la protection efficace et durable de cette culture contre les nématodes. Il s'agit spécifiquement d'identifier les espèces de nématodes recensés, d'évaluer l'efficacité de la fertilisation organo-minérale sur les nématodes et d'évaluer le rendement en rhizome frais du gingembre.

\section{Matériel et méthodes \\ Site expérimental}

L'étude a été menée à la Station d'Expérimentations Agronomiques de 1'Ecole Supérieure d'Agronomie, à l'Université de Lomé (06 $17^{\circ} \mathrm{N}$, $\left.001^{\circ} 21^{\prime} \mathrm{E}\right)$. Le climat est tropical de type guinéen avec une pluviométrie annuelle variant de 800 à $1200 \mathrm{~mm}$. Le sol de type ferralitique, est qualifié de «terre de barre». La composition chimique est de $1,87 \%$ de matière organique, $0,15 \%$ d'Azote $(\mathrm{N})$ total, 0,05\% de Phosphore $\left(\mathrm{P}_{2} \mathrm{O}_{5}\right), 0,46$ de Potassium $\left(\mathrm{K}_{2} \mathrm{O}\right)$ et de 0,01 de Magnésium $(\mathrm{MgO})$ et celle des bases échangeables en $\mathrm{mg} / \mathrm{Kg}$ est de : $51,1 \mathrm{Mg}^{2+} ; 210,1 \mathrm{Ca}^{2+} ; 148,8 \mathrm{~K}^{+}$et $15,3 \mathrm{Na}^{+}$. Le potentiel hydrogène $\mathrm{pH}$ est de 6,8 . Le site expérimental est naturellement infesté par des populations mixtes de nématodes. 


\section{Matériel végétal}

Le gingembre jaune a servi de matériel végétal pour cette étude. Les rhizomes ont été achetés chez des producteurs dans la région des plateaux précisément à Kpalimé. Il est produit localement et a un cycle de 6 à 8 mois.

\section{Fumure minérale et organique}

Cinq formulations à base d'engrais simples (urée $\left(\mathrm{CO}\left(\mathrm{NH}_{2}\right)_{2}\right)$ dosant $46 \%$ de N, triple superphosphate (TSP) dosant $46 \%$ de $\mathrm{P}_{2} \mathrm{O}_{5}$ et chlorure de potassium $(\mathrm{KCl})$ dosant $60 \%$ de $\mathrm{K}_{2} \mathrm{O}$ ) ont été utilisées.

La fumure organique est produite avec des déchets ménagers et des déjections animales (fientes, bouses, etc.). Elle a été achetée auprès de la société ENPRO (Ecosystème Naturel Propre). Les teneurs en $\mathrm{N}$, en $\mathrm{P}_{2} \mathrm{O}_{5}$, en $\mathrm{K}_{2} \mathrm{O}$ et en matière organique selon le fournisseur sont supérieures à $1,5 \%$, $2,5 \%, 0,8 \%$ et $40 \%$ respectivement.

\section{Dispositif expérimental et conduite de l'essai}

L'expérimentation a été conduite pendant deux saisons, 2015-2016 et 2016-2017 suivant un dispositif expérimental en split-plot avec trois répétitions et 15 traitements. L'unité expérimentale est constituée d'une parcelle de 2,5 $\mathrm{m}$ de long sur $1 \mathrm{~m}$ de large, soit 2,5 $\mathrm{m}^{2}$. Les traitements sont définis par la combinaison de deux facteurs : la dose de fumure organique à trois niveaux F0 $\left(0\right.$ t.ha $\left.^{-1}\right), \mathrm{F} 1\left(15\right.$ t.ha $\left.^{-1}\right)$ et $\mathrm{F} 2\left(25\right.$ t.ha $\left.^{-1}\right)$ et la fumure minérale avec cinq formulations : $\mathrm{M} 0\left(\mathrm{~N}_{0} \mathrm{P}_{0} \mathrm{~K}_{0}\right)$, M1 $\left(\mathrm{N}_{45} \mathrm{P}_{15} \mathrm{~K}_{60}\right), \mathrm{M} 2\left(\mathrm{~N}_{70} \mathrm{P}_{50} \mathrm{~K}_{50}\right), \mathrm{M} 3$ $\left(\mathrm{N}_{90} \mathrm{P}_{30} \mathrm{~K}_{100}\right)$ et $\mathrm{M} 4\left(\mathrm{~N}_{125} \mathrm{P}_{90} \mathrm{~K}_{100}\right)$. L'engrais minéral est en sous-parcelles et la fumure organique en parcelle principale. La fumure organique a été apportée au moment du labour. Quant aux engrais minéraux ( $\mathrm{KCl}$ et TSP), ils ont été apportés 40 jours après semis. Cependant l'urée $\left(\mathrm{CO}\left(\mathrm{NH}_{2}\right)_{2}\right)$, est fractionnée en deux apports : 40 jours et 110 jours après le semis. La récolte a eu lieu sept mois après semis. Des entretiens (sarclage, binage, épandage d'engrais, etc.) réguliers ont été faits au besoin tout au long du cycle.

\section{Estimation des densités de populations des nématodes}

Les effets des différents traitements sur les densités nématodes ont été évalués en estimant les densités de populations de nématodes dans le sol et dans les racines six mois après semis, correspondant à l'entrée en senescence de la plante. Sur chaque parcelle élémentaire, trois plants sur de la ligne centrale ont été prélevé au hasard. Les racines provenant des plants d'une même parcelle ont été soigneusement lavées à l'eau de robinet et mélangées. Ces racines ont été par la suite découpées à l'aide d'une paire de ciseaux en petits morceaux d'un centimètre en moyenne et ont été broyées au moyen d'un mixeur. Les nématodes ont été extraits dans $5 \mathrm{~g}$ de chaque échantillon de racines en utilisant la technique de broyage (Coyne et al., 2010). 
Quant au sol, les prélèvements ont été effectués approximativement à $20 \mathrm{~cm}$ de profondeur (Hooper et al., 2005) des plants sélectionnés. Les échantillons d'une même parcelle ont été mélangés et bien malaxés pour obtenir un échantillon représentatif. Les nématodes telluriques ont été extraits en utilisant la technique modifiée de Baermann dans $100 \mathrm{~g}$ de sol (Coyne et al., 2010). Chaque échantillon ainsi préparé a été mis dans un tamis tapissé à l'intérieur par du papier hygiénique (jouant le rôle de filtre) et le tout est placé dans une bassine en plastique. Ensuite l'eau a été ajoutée jusqu'à couvrir légèrement l'échantillon de sols ou de racines pour favoriser la migration des nématodes des échantillons de sols et de racines vers l'eau qui constitue le milieu d'extraction. Après 24 heures pour le sol et 48 heures pour les racines, le tamis contenant le papier filtre sur lequel est déposé l'échantillon est retiré délicatement de la bassine. L'eau du bol contenant les nématodes est recueillie dans des tubes gradués et après 30 minutes de décantation, l'observation a été faite. La densité des nématodes a été évalué dans un volume de $100 \mathrm{ml}$ de l'extrait. Les effectifs de la population exprimés sont des moyennes de trois observations à la loupe binoculaire dans des prélèvements de l'extrait (Bissadou et al., 2012).

\section{Fixation et identification des nématodes}

La fixation des nématodes a consisté à ajouter à volume égale la suspension de nématodes à une solution fixatrice chauffée modérément. Cette solution fixatrice est composée de: triethanolamine $2 \%$, formaline (formaldéhyde à 35\%) $8 \%$ et de l'eau distillée 90\% (Coyne et al., 2010). La solution de fixation reste stable pour une très longue période et les nématodes gardent une apparence proche de leur vivant car ils ne se dessèchent pas. Ces échantillons fixés ont été envoyé à l'Université de Gand (Belgique) pour identification.

\section{Evaluation du rendement des rhizomes frais}

Sept mois après la mise en place, les rhizomes frais de toutes les parcelles élémentaires ont été déterrés puis pesés au moyen d'une balance. Mais avant d'être pesés, ils ont été débarrassés de tous débris végétaux et mottes de terres.

\section{Analyse statistique}

Toutes les données ont été analysées à l'aide du logiciel GenStat Discovery Edition 4 par une analyse de la variance (ANOVA) et les moyennes sont discriminées à l'aide du Test de Student Newman-Keuls au seuil de 5\%. La densité des nématodes est transformée en $X^{\prime}=\log 10(X+1)$ avant l'analyse pour normaliser les données, $\mathrm{X}$ étant le nombre de nématodes comptés (Gomez \& Gomez, 1984). 


\section{Résultats}

\section{Identification des différentes espèces de nématodes recensées}

L'inventaire global réalisé sur les différents échantillons du sol et des racines (tableau I), a fait ressortir un effectif total de 796 individus appartenant à 5 genres. Il s'agit des genres Meloidogyne; Xiphinema; Longidorus ; Helicotylenchus et Pratylenchus. Dans ce total de 796 individus recensés, le genre Meloidogyne représente à lui seul 64,77\% de la population de nématodes identifiés dans le sol et $99,43 \%$ des nématodes identifiés dans les racines. Les nématodes racinaires ont été plus importants en termes d'effectif (708 contre 88 ) ; cependant on note une plus grande diversité au niveau du sol (04 genres contre 02 genres).

Tableau I : Liste systématique et fréquences d'abondances (\%) des nématodes recensés dans le sol et dans les racines du gingembre

\begin{tabular}{cccccc}
\hline & & & \multicolumn{2}{c}{ Fréquence d'abondance (\%) } \\
\cline { 3 - 6 } Classe & Ordre & Famille & Genre & $\begin{array}{c}\text { Nématodes du } \\
\text { sol (N=88) }\end{array}$ & $\begin{array}{c}\text { Nématodes } \\
\text { racinaires (N=708) }\end{array}$ \\
\hline \multirow{5}{*}{ Secernentea } & Tylenchida & Haplolaimidae & Pratylenchus & & 0,50 \\
& & Heteroderidae & Meloidogyne & 64,77 & 99,43 \\
& & Hoplolaimidae & Helicotylenchus & 13,63 & \\
\cline { 2 - 6 } & Dorylaimida & Longidoridae & Longidorus & 01,10 & 0 \\
& & & Xiphinema & 20,45 & 0 \\
\hline
\end{tabular}

\section{Effets des fumures organique et minérale sur la densité des nématodes}

A l'extraction (six mois après semis), les densités moyennes de populations des nématodes ont significativement varié entre les différents traitements aussi bien au niveau du sol $(\mathrm{P}<0,001)$ que dans les racines $(\mathrm{P}<$ 0,001) (Tableau II).

Au niveau des racines, la faible densité des nématodes est obtenue avec l'apport de 25 tonnes de fumure organique associé à la fumure minérale $\mathrm{N}_{70} \mathrm{P}_{50} \mathrm{~K}_{50}$. Cette combinaison a permis de réduire de plus de 73,84\% la densité des nématodes par rapport au témoin. Par rapport aux nématodes telluriques, c'est plutôt 1'apport de 25 tonnes de fumure organique associé à la fumure minérale $\mathrm{N}_{125} \mathrm{P}_{90} \mathrm{~K}_{100}$ qui a donné le meilleur résultat. Que ce soit au niveau $\mathrm{du}$ sol ou au niveau des racines, l'association fumure organique - fumure minérale a semblé avoir un meilleur effet que l'apport de la fumure minérale seule.

Tableau II : Effets de la dose de fumure organique et des différentes formulations à base de $\mathrm{N}, \mathrm{P}$ et $\mathrm{K}$ sur la densité des nématodes inféodés à la culture du gingembre

\begin{tabular}{lcc}
\hline \multirow{2}{*}{ Formulations d'engrais } & \multicolumn{2}{c}{ Densité moyenne des nématodes à 6 mois* } \\
\cline { 2 - 3 } & Racine & \multicolumn{1}{c}{ Sol } \\
\hline F0M0 $\left(0 \mathrm{t} / \mathrm{ha}+\mathrm{N}_{0} \mathrm{P}_{0} \mathrm{~K}_{0}\right)$ & $29,33 \mathrm{a}$ & $191,70 \mathrm{a}$ \\
F0M1 $\left(0 \mathrm{t} / \mathrm{ha}+\mathrm{N}_{45} \mathrm{P}_{15} \mathrm{~K}_{60}\right)$ & $21,00 \mathrm{ab}$ & $168,00 \mathrm{a}$ \\
F0M2 $\left(0 \mathrm{t} / \mathrm{ha}+\mathrm{N}_{70} \mathrm{P}_{50} \mathrm{~K}_{50}\right)$ & $24,00 \mathrm{ab}$ & $85,30 \mathrm{bc}$ \\
F0M3 $\left(0 \mathrm{t} / \mathrm{ha}+\mathrm{N}_{90} \mathrm{P}_{30} \mathrm{~K}_{100}\right)$ & $10,67 \mathrm{cde}$ & $110,30 \mathrm{ab}$
\end{tabular}




\begin{tabular}{lll} 
F0M4 (0t/ha $\left.+\mathrm{N}_{125} \mathrm{P}_{90} \mathrm{~K}_{100}\right)$ & $22,00 \mathrm{ab}$ & $41,30 \mathrm{efg}$ \\
F1M0 $\left(15 \mathrm{t} / \mathrm{ha}+\mathrm{N}_{0} \mathrm{P}_{0} \mathrm{~K}_{0}\right)$ & $10,00 \mathrm{de}$ & $68,70 \mathrm{bcd}$ \\
F1M1 $\left(15 \mathrm{t} / \mathrm{ha}+\mathrm{N}_{45} \mathrm{P}_{15} \mathrm{~K}_{60}\right)$ & $22,33 \mathrm{ab}$ & $63,70 \mathrm{cde}$ \\
F1M2 $\left(15 \mathrm{t} / \mathrm{ha}+\mathrm{N}_{70} \mathrm{P}_{50} \mathrm{~K}_{50}\right)$ & $15,00 \mathrm{bcd}$ & $39,30 \mathrm{efg}$ \\
F1M3 $\left(15 \mathrm{t} / \mathrm{ha}+\mathrm{N}_{90} \mathrm{P}_{30} \mathrm{~K}_{100}\right)$ & $09,00 \mathrm{de}$ & $34,70 \mathrm{fgh}$ \\
F1M4 (15t/ha $\left.+\mathrm{N}_{125} \mathrm{P}_{90} \mathrm{~K}_{100}\right)$ & $22,67 \mathrm{ab}$ & $48,30 \mathrm{def}$ \\
F2M0 (25t/ha $\left.+\mathrm{N}_{0} \mathrm{P}_{0} \mathrm{~K}_{0}\right)$ & $20,00 \mathrm{ab}$ & $37,70 \mathrm{fgh}$ \\
F2M1 $\left(25 \mathrm{t} / \mathrm{ha}+\mathrm{N}_{45} \mathrm{P}_{15} \mathrm{~K}_{60}\right)$ & $20,67 \mathrm{ab}$ & $41,30 \mathrm{fgh}$ \\
F2M2 (25t/ha $\left.+\mathrm{N}_{70} \mathrm{P}_{50} \mathrm{~K}_{50}\right)$ & $07,67 \mathrm{e}$ & $78,70 \mathrm{bc}$ \\
F2M3 (25t/ha $\left.+\mathrm{N}_{90} \mathrm{P}_{30} \mathrm{~K}_{100}\right)$ & $18,33 \mathrm{abc}$ & $24,70 \mathrm{gh}$ \\
F2M4 (25t/ha $\left.+\mathrm{N}_{125} \mathrm{P}_{90} \mathrm{~K}_{100}\right)$ & $11,33 \mathrm{bcde}$ & $22,00 \mathrm{~h}$ \\
CV $(\%)$ & 10,80 & 07,90 \\
P & $<0,001$ & $<0,001$ \\
\hline
\end{tabular}

*Les valeurs sont les moyennes de la densité des nématodes des trois répétitions

Les moyennes dans la même colonne suivies de la même lettre sont statistiquement identiques selon le test de Newman-Keuls à 5\%.

\section{Effets des engrais sur le rendement en rhizomes frais}

Les rendements en rhizomes frais sous l'effet des engrais minéraux et de la fumure organique sont consignés dans le tableau III. Ces résultats montrent que l'apport des engrais a entraîné une augmentation des rendements quoi que statistiquement non significatif. Le plus grand rendement $(7,73 \mathrm{t} / \mathrm{ha})$ est obtenu avec l'association de $25 \mathrm{t} / \mathrm{ha}$ de fumure organique avec la fumure minérale $\mathrm{N}_{125} \mathrm{P}_{90} \mathrm{~K}_{100}$. Cet apport a permis une augmentation de plus $163 \%$ du rendement comparativement au témoin sans fumure.

Tableau III : Effets de la dose de fumure organique et des différentes formulations à base de $\mathrm{N}, \mathrm{P}$ et $\mathrm{K}$ sur le rendement en rhizomes frais du gingembre

\begin{tabular}{ll}
\hline Formulation engrais & Rendement rhizomes frais (t/ha) \\
\hline F0M0 $\left(0 t / h a+\mathrm{N}_{0} \mathrm{P}_{0} \mathrm{~K}_{0}\right)$ & 2,93 \\
F0M1 $\left.\left(0 t / h a+\mathrm{N}_{45} \mathrm{P}_{15} \mathrm{~K}_{60}\right)\right)$ & 3,40 \\
F0M2 $\left(0 t / h a+\mathrm{N}_{70} \mathrm{P}_{50} \mathrm{~K}_{50}\right)$ & 4,20 \\
F0M3 $\left(0 t / h a+\mathrm{N}_{90} \mathrm{P}_{30} \mathrm{~K}_{100}\right)$ & 3,70 \\
F0M4 $\left(0 t / h a+\mathrm{N}_{125} \mathrm{P}_{90} \mathrm{~K}_{100}\right)$ & 4,27 \\
F1M0 $\left(15 t / h a+\mathrm{N}_{0} \mathrm{P}_{0} \mathrm{~K}_{0}\right)$ & 3,80 \\
F1M1 $\left(15 t / h a+\mathrm{N}_{45} \mathrm{P}_{15} \mathrm{~K}_{60}\right)$ & 4,53 \\
F1M2 $\left(15 t / h a+\mathrm{N}_{70} \mathrm{P}_{50} \mathrm{~K}_{50}\right)$ & 5,07 \\
F1M3 $\left(15 t / h a+\mathrm{N}_{90} \mathrm{P}_{30} \mathrm{~K}_{100}\right)$ & 5,60 \\
F1M4 $\left(15 t / h a+\mathrm{N}_{125} \mathrm{P}_{90} \mathrm{~K}_{100}\right)$ & 4,20 \\
F2M0 $\left(25 t / h a+\mathrm{N}_{0} \mathrm{P}_{0} \mathrm{~K}_{0}\right)$ & 4,00 \\
F2M1 $\left(25 t / h a+\mathrm{N}_{45} \mathrm{P}_{15} \mathrm{~K}_{60}\right)$ & 3,53 \\
F2M2 $\left(25 t / h a+\mathrm{N}_{70} \mathrm{P}_{50} \mathrm{~K}_{50}\right)$ & 5,73 \\
F2M3 $\left(25 t / h a+\mathrm{N}_{90} \mathrm{P}_{30} \mathrm{~K}_{100}\right)$ & 6,93 \\
F2M4 $\left(25 t / h a+\mathrm{N}_{125} \mathrm{P}_{90} \mathrm{~K}_{100}\right)$ & 7,73 \\
CV & 31,60
\end{tabular}


$\mathrm{P}(5 \%)$

0,31

Les valeurs sont les moyennes des trois répétitions des différents traitements

Cependant à la récolte, on a remarqué une infestation plus ou moins homogène des rhizomes par les nématodes qui se traduit par la pourriture des racines des rhizomes (Figure 1).

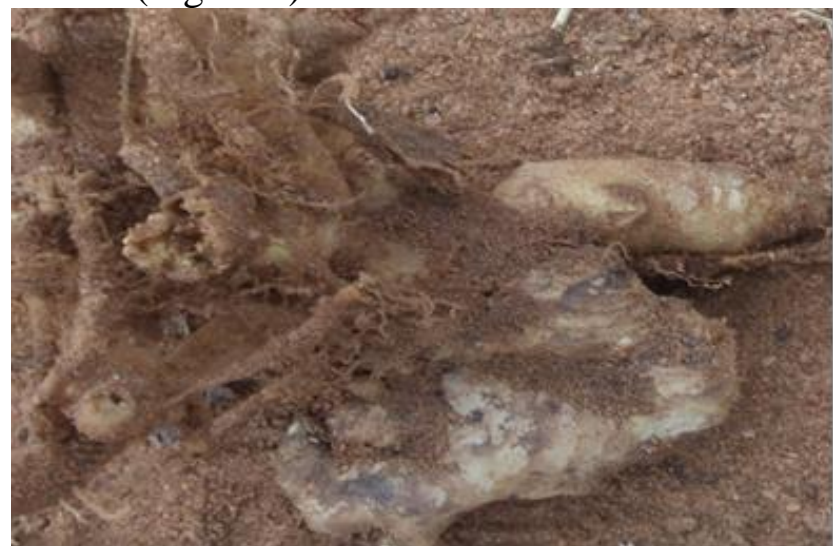

Figure 1 : rhizome de gingembre avec ses racines pourries dû aux attaques des nématodes

\section{Discussion}

L'identification des nématodes nous a permis de recenser cinq genres sur le site de culture. Il s'agit de Meloidogyne, Xiphinema, Helicotylenchus, Pratylenchus et Longidorus. Parmi les genres recensés, on a trois ectoparasites (Xiphinema, Longidorus et Helicotylenchus); un endoparasite migratoire (Pratylenchus) et un endoparasite sédentaire (Meloidogyne). Parmi ces genres inventoriés, Meloidogyne représente à elle seule plus de $95 \%$ du nombre total de nématodes identifiés. Ceci corrobore les résultats des études de Sikora \& Fernandez (2005) ; Fanou et al. (2005) qui rapportent que les nématodes à galles (Meloidogyne) semblent être le principal problème des cultures maraichères en Afrique de l'Ouest.

Cependant, il faut souligner que ces genres recensés ne sont pas forcément liés au gingembre mais sont généralement polyphages. En outre, ceci peut être lié au type de sol. La relation (nématode-type de sol), nommée relation mésologique, est connue depuis longtemps (Cadet, 1998). Baimey et al. (2009) ont observé que la répartition des nématodes phytoparasites est en relation avec le sol. Ainsi la présence d'une plante ne déterminant pas obligatoirement celle des espèces de nématodes qui sont capables de la parasiter. De plus, Cadet et al. (2005), notent que les caractéristiques du sol affectent l'abondance, la distribution et la structure des communautés de nématode, indépendamment de l'influence directe de la plante hôte.

L'apport d'engrais organique associé à la fumure minérale a permis de réduire la densité des nématodes comparativement au témoin n'ayant pas reçu 
d'apport d'engrais. Ces résultats montrent que la fertilisation a permis de réduire significativement la densité des nématodes aussi bien au niveau du sol et que des racines. Mais, la densité des nématodes au niveau des plantes qui ont reçu uniquement que la fumure minérale ou la fumure organique est plus élevée que celles ayant reçu la combinaison des deux. Ceci démontre le potentiel de la combinaison fumure organique et fumure minérale comme solution efficace pour lutter contre les nématodes en culture du gingembre. Des résultats similaires ont été rapportés par certaines études antérieures qui montrent que l'application de la fumure organique (El Gharras et al., 2011 ; Bissadou et al., 2012) ou de la fumure minérale (Traore et al., 2014) ou les deux (Traore et al., 2012 ) permet de réduire de façon significative la population des nématodes et d'augmenter le rendement. Ces résultats peuvent s'expliquer par les phénomènes d'allélopathie soit directe (production de composées chimiques toxiques, inhibiteurs, répulsifs des nématodes par la plante) soit indirecte (fourniture de niches pour l'installation de flore et de faune antagonistes) (Bais et al., 2006). Il est également rapporté que les plantes bien nourries tolèrent mieux les attaques des nématodes dus à la compensation des dégâts infligés à la plante via l'amélioration de la fertilité du sol et de la disponibilité des nutriments (Tabarant, 2011). De plus la faible densité des nématodes recensée au niveau des racines par rapport au sol peut être en lien avec la structure des racines et laisse penser à un phénomène physique.

La densité des nématodes dans le sol a tendance à être faible avec les doses croissantes d'azote $(\mathrm{N})$. En effet, il a été démontré qu'une grande quantité d'azote dans le sol serait responsable de la plasmolyse de nématodes, mais aussi de la prolifération de champignons nématophages et de champignons producteurs de chitinase qui s'attaquent à la coquille des œufs des nématodes (Rodriguez-Kabana, 1986).

Bien que l'apport des engrais a tendance à entraîner une augmentation des rendements en rhizomes frais du gingembre due à une plus grande disponibilité en éléments nutritifs pour les plants, nos rendements restent faibles et statistiquement non significatifs. Cette tendance d'augmentation de rendement due aux apports des engrais est similaire à celle de certaines études antérieures notamment celles de Haque et al. (2007), d'Akhter et al. (2013) et de Singh et al. (2016) qui recommandent respectivement $\mathrm{N}_{120} / \mathrm{ha}, \mathrm{K}_{120} /$ ha et $\mathrm{N}_{180} \mathrm{~K}_{160}$ pour obtenir un bon rendement ou de celle d'Ajithkumar \& Jayachandran (2001) qui rapportent qu'il faut des doses d'au moins $\mathrm{N}_{150} \mathrm{P}_{100} \mathrm{~K}_{100}$ par hectare pour donner de bon rendement dans le cas d'une culture associée gingembre et noix de coco au Nigeria.

Les attaques des nématodes n'ont pas permis d'avoir des rendements statistiquement significatifs. En outre, au cours du cycle, des attaques de nématodes plus ou moins sévères recensées sur les parcelles ont entrainé une baisse drastique des rendements. Ces derniers attaquent des plants de 
gingembre et entrainent la formation de galles, la pourriture des racines et rendent les plants vulnérables à d'autres ravageurs et maladies (Coyne et al., 2010). Ces attaques de nématodes réduisent la potentialité des plants à utiliser efficacement les nutriments apportés. On peut également penser à un apport d'engrais insuffisant, car selon Lujiu et al. (2004), une culture de gingembre est en mesure d'exporter jusqu'à $400 \mathrm{~kg} / \mathrm{ha}$ de $\mathrm{N}, 32 \mathrm{~kg} / \mathrm{ha}$ de $\mathrm{P}$ et $394 \mathrm{~kg} / \mathrm{ha}$ de $\mathrm{K}$.

La grande variabilité obtenue dans les résultats de rendement confirme effectivement l'infestation naturelle de la parcelle par les nématodes. En effet, notre expérimentation a été effectuée sur une parcelle infestée de façon naturelle. Les résultats du dénombrement des nématodes montrent effectivement une inégale répartition des nématodes sur les parcelles. Cette inégale répartition est due à des compétitions interspécifiques ou une inégale répartition des certains éléments physico-chimiques du sol (Cadet, 1998 ; Traore et al., 2012).

\section{Conclusion}

Les nématodes constituent un grave problème phytosanitaire en cultures maraichères. Dans le cadre de la recherche des méthodes alternatives à l'utilisation des produits chimiques pour contrôler les nématodes une première étude sur le gingembre a été réalisée au Togo. Les résultats ont montré que l'apport d'engrais organique associé à la fumure minérale permet de réduire la densité des nématodes racinaires et telluriques. Le meilleur rendement est obtenu avec l'apport de 25 t/ha de fumure organique associée à la fumure minérale $\mathrm{N}_{125} \mathrm{P}_{90} \mathrm{~K}_{100}$ de fumure minérale soit 7,3 tha ${ }^{-1}$. Quant à la némato-régulation par fertilisation, l'association fumure minérale-fumure organique semble être la meilleure option. Cet apport permet d'augmenter le rendement en rhizomes frais du gingembre. L'étude a également révélé que les nématodes détruisent les systèmes racinaires et empêchent l'alimentation de la plante. Parmi les espèces de nématodes recensées, Melodogyne spp est la plus abondante dans la zone de l'étude. Des travaux doivent continuer dans les zones agro-écologiques en vue d'une vulgarisation.

\section{References:}

1. Ajithkumar, K. \& Jayachandran, B. K. (2001). Effect of major nutrients on yield of ginger (Zingiber officinale Rose.) intercropped in coconut garden. Journal of Spices and Aromatic Crops 10: 17-23.

2. Akhter, S., Noor, S., Islam, M., Masud, M., Talukder, M. \& Hossain, M. (2013). Effect of Potassium Fertilization on the Yield and Quality of Ginger (Zingiber officinale) grown on a K Deficient Terrace Soil of Level Barind Tract (AEZ 25) in Northern Bangladesh. e-ifc 35, September 2013. 
3. Bais, H., Weir, T., Perry, L., Gilroy, S. \& Vivanco, J. (2006). The role of root exudates in rhizosphere interactions with plants and other organisms. Annual review of plant biology 5: 233-266.

4. Baimey, H., Coyne, D., Dagbenonbakin, G. \& James, B. (2009). Plantparasitic nematodes associated with vegetable crops in Benin: relationship with soil physico-chemical properties. Nematologia Mediterranea 37: 225-234.

5. Bissadou, K. D., Tchabi, A., Tounou, A. K., Ayessom, A. \& Gumedzoe, M. (2012). Impact de la fumure organique appliquée seule et en combinaison avec une souche indigène de champignon mycorhizien arbusculaire Glomus mosseae sur Meloidogyne spp, principal nématode parasitaire de la tomate au Togo. Journal of Applied Biosciences 55: 3973- 3986.

6. Cadet, P. (1998). Gestion écologique des nématodes phytoparasites tropicaux. Cahier d'Agriculture 7: 187-194.

7. Cadet, P., Masse, D. \& Thioulouse, J. (2005). Relationships between plant-parasitic nematode community, fallow duration and soil factors in the Sudano-Sahelian area of Senegal. Agriculture, Ecosystems and Environment, 102: 302-317.

8. Coyne, D. L., Nicol, J. M. \& Claudius-Cole, B. (2010). Les nématodes des plantes : Un guide pratique des techniques de terrain et de laboratoire. Secrétariat SP-IPM, Institut International d'Agriculture Tropicale (IITA), Cotonou, Benin. 93p.

9. Egbuchua, C. N. \& Enujeke, E. C. (2013). Growth and yield responses of ginger (Zingiber officinale) to three sources of organic manures in a typical rainforest zone, Nigeria. Journal of Horticulture and Forestry 5: 109-114.

10. El Badri, G., Lee, D., Park, J., Bin Yu, H. \& Choo, H., (2008). Evaluation of various plant extracts for their nematicidal efficacies against juveniles of Meloidogyne incognita. Journal of Asia-Pacific Entomology 11: 99-102.

11. Eleazu, C.O., Amadi, C.O., Iwo, G., Nwosu, P. \& Ironua, C. (2013). Chemical Composition and Free Radical Scavenging Activities of 10 Elite Accessions of Ginger (Zingiber officinale Roscoe). Journal of Clinic Toxicology 3: 155.

12. El Gharras, Y., Bouzoubaâ, Z. \& Ait Lhaj, A. (2011). Comparaison de l'effet fertilisant et contrôle des nématodes des tourteaux de neem, tourteau de ricin, tourteau d'argan et feuilles d'argan sur une culture de courgette en conduite biologique. Actes du Premier Congrès International de 1'Arganier, Agadir: 442-447.

13. Fanou, A., Glitho, M., Baimey, H. \& Sagbohan, J. (2005). Étude comparée des pesticides botaniques sur les organismes nuisibles des 
cultures maraîchères (carotte, oignon et gboma) dans les centres maraîchers d'Agron de Sokotomey, de Grand popo et d'Adjohoun. In : Recherche Agricole pour le développement. Actes de l'Atelier.

14. Gomez, K. A. \& Gomez, A. A. (1984). Statistical Procedures for Agricultural Research. 2 édition, John Wiley and Sons Inc., NewYork, USA. 680pp.

15. Haque, M. M., Rahman, A. K. M. M., Ahmed, M., Masud, M. M. \& Sarker, M. M. R. (2007). Effect of Nitrogen and Potassium on the Yield and Quality of Ginger in Hill Slope. Journal Soil Nature 1: 0510.

16. Hooper, D. J., Hallmann, J. \& Subbotin, S. (2005). Methods for extraction, processing and detection of plant and soil nematodes: 5386. In: Luc, M., Sikora, R. A., Bridge, J., (eds), Plant Parasitic Nematodes in Subtropical and Tropical Agriculture. Wallingford,UK.

17. James, B., Atcha-Ahowé, C., Godonou, I., Baimey, H., Goergen, H., Sikirou, R. \& Toko, M. (2010). Integrated pest management in vegetable production: A guide for extension workers in West Africa. International Institute of Tropical Agriculture, Ibadan, Nigeria. 114pp.

18. Koshy, P. K, Santhoch, J. E. \& Rakesh, P. (2005). Nematode Parasites of Spices, Condiments and Medicinal Plants.

19. Lujiu, L., Xisheng, G., Jiejun, G., Nan, D. \& Lin, Z. (2004). Ginger Response to Potassium in Anhui Province. Better Crops 88: 22-24.

20. Manzanilla-Lopez, R. H., Kenneth, E. \& Bridge, J. (2004). Plant diseases caused by nematodes: 637-716. In: Chen, Z. X., Chen, S. Y. \& Dickson, D. W. (eds), Nematology - Advances and Perspectives. Nematode Management and Utilization. Wallingford, UK.

21. Nandkangre, H., Ouedraogo, M., \& Sawadogo, M. (2015). Caractérisation du système de production du gingembre (Zingiber officinale Rosc.) au Burkina Faso : Potentialités, contraintes et perspectives. International of Journal of Biology Chemistry Science 9: 861-873.

22. Preeti, C., Dnyaneshwar, W., Kalpana, J. \& Bhushan, P. (2008). Development of SCAR (sequence-characterized amplified region) markers as a complementary tool for identification of ginger (Zingiber officinale Roscoe) from crude drugs and multi component formulations. Biotechnology Applied Biochemestry 50: 61-69.

23. Rodriguez-Kabana, R. (1986). Organic and inorganic nitrogen amendments to soil as nematode suppressants. Journal of Nematology 18: 129.

24. Seinhorst, J. (1956). Population studies on stem eelworms (Ditylenchus dipsaci). Nematologica 1: 159-64. 
25. Singh, M., Masroor, M. \& Naeem, M. (2016). Effect of nitrogen on growth, nutrient assimilation, essential oil content, yield and quality attributes in Zingiber officinale Rosc. Journal of the Saudi Society of Agricultural Sciences 15: 171-178.

26. Sikora, R. A. \& Fernández, E. (2005). Nematode Parasites of Vegetables In: M. Luc, R. A. Sikora et J. Bridge (Eds.). Plant Parasitic Nematodes in Subtropical and Tropical Agriculture. CAB International, Wallingford, Oxon, UK. Pp. 319 - 392.

27. Tabarant, P. (2011). Effets d'apport de matières organiques sur le contrôle biologique des nématodes parasites du bananier en Guadeloupe. Thèse de Doctorat, Institut des Sciences et Industries du Vivant et de l'Environnement. 176p.

28. Traore, M., Lompo, F., Thio, B., Ouattara, B., Ouattara, K. \& Sedogo, M. (2012). Influence de la rotation culturale avec apport de matières organiques exogènes et d'une fertilisation minérale sur les nématodes phytoparasites en culture du sorgho au Centre Ouest du Burkina Faso. International Journal Biological and Chemical Sciences 6: 628-640.

29. Traore, M., Lompo, F., Thio, B., Ouattara, B., Ouattara, K. \& Sedogo, M. (2014). Etude des nematodes phytoparasites du sol et des raciness sous quatres niveaux de fertilization minérale en culture de niébé. Int J. Biol. Chem. Sci. 8(3), pp. 891-902.

30. Trudgill, D. L. \& Blok, V.C. (2001). Apomictic, polyphagous rootknot nematodes: exceptionally successful and damaging biotrophic root pathogens. Annual Review Phytopathology 39: 53-77. 